\title{
On the boundary creation of the isogeometric-BEM in two dimensional topology optimization based on the level-set method
}

\author{
Peiwen MIAO $^{1)}$, Toru TAKAHASHI ${ }^{1)}$, \\ Hiroshi ISAKARI $^{1)}$, Toshiro MATSUMOTO ${ }^{1)}$ \\ ${ }^{1)}$ Nagoya University, Furo-cho, Chikusa-ku, Nagoya 464-8603, Japan
}

Key Words : Isogeometric analysis, Level-set method, D-curvature, Self-intersection

The isogeometric boundary element method (IGBEM) has been successfully applied to shape optimization [1], while for topology optimization there still exist some issues to be solved. The main challenge in applying IGBEM to topology optimization based on the level-set method locates on the creation of the boundary from the level-set function with the B-spline curves. This talk shows a way to create a boundary that is smooth and non-oscillating. In addition, we will propose an algorithm to resolve the issue of self-intersection of the created boundary.

To introduce IGBEM to level-set based topology optimization, it is necessary to generate B-spline boundary from the zero contour line of level-set function. Each contour is piecewise-linear and we call the nodes of the contour as zero points.

In order to approximate an contour with a B-spline curve, we need to determine the initial position of the control points (CPs), where the number of CPs is less than that of zero points. To this end, we utilize the discrete curvature (D-curvature) [2] of a piecewise-linear curve. The D-curvature of node $i$, denoted by $D_{i}$, is defined as:

$$
D_{i}=\frac{\sigma_{i}}{R_{i}}=\frac{2 \sigma_{i} \Delta_{i}}{l_{i} l_{i+1} Q_{i}},
$$

where $R_{i}$ denotes the radius of circumcirle of triangle $T_{i}$, consists of $\boldsymbol{z}_{i-1}, \boldsymbol{z}_{i}$, and $\boldsymbol{z}_{i+1}, l_{i}, l_{i+1}$ and $Q_{i}$ denotes three sides of $T_{i}$ (see fig.1). Also, $\sigma_{i}=1$ if the order of $\boldsymbol{z}_{i-1}, \boldsymbol{z}_{i}$, and $\boldsymbol{z}_{i+1}$ is clockwise and $\sigma_{i}=-1$ otherwise.

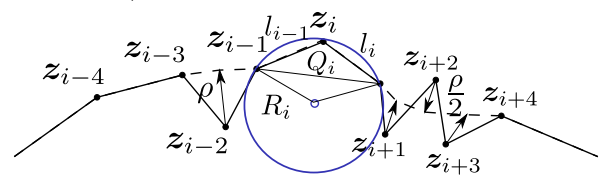

Fig. 1 The discrete curvature and smoothing of piecewise-linear curve

Then, we treat node $\boldsymbol{z}_{i}$ satisfying $D_{i-1} D_{i}<0$ and $D_{i+1} D_{i}<0$ as bad node and move it to the gravity center of triangle $T_{i}$ with a presetted distance $\rho$. Specifically, regarding a node whose neighbours are also bad, we move the node by $\rho / 2$. In this talk, $\rho$ is given as half of the average side length of the triangular finite element we use.

Next, we compute local-curvature-maximum (LCM) points, which satisfies $D_{i}>D_{i+1}$ and $D_{i}>D_{i-1}$. We select LCM points as well as some points between LCM points when those points are necessary for ensuring the accuracy of IGBEM. We then fit the B-spline curve associated with the control points to the underlying piecewiselinear curve so that the following objective function $f$ is minimized by L-BFGS [3]:

$$
f(\mathcal{C})=\frac{1}{2} \sum_{i=1}^{n}\left\|\boldsymbol{p}\left(\mathcal{C}, t_{i}\right)-\boldsymbol{z}_{i}\right\|^{2},
$$

where $\mathcal{C}$ stands for the set of control points. $\boldsymbol{p}$ denotes the points corresponding to $\mathcal{C}$ and curve parameter $t_{i}$

We then propose an anlgorithm to resolve the issue of self-intersection that could happen after approximation. Basically, if a segment $l_{i}$, which is the segment between control points $\boldsymbol{c}_{i}$ and $\boldsymbol{c}_{i+1}$, and $l_{j}$ is intersected, we invert the order of $c_{i+1} \cdots c_{j}$ like the simple example in fig. 2 .
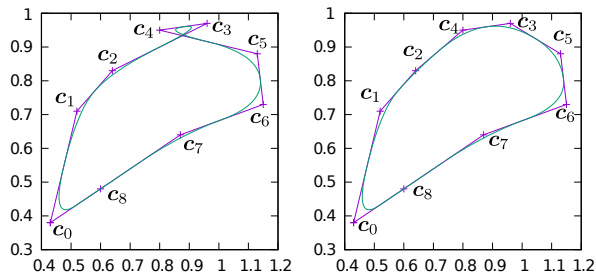

Fig. 2 Resolving self-intersection, purple line denotes the control polygon and $B$-spline curve is shown in green color

The details of our proposed algorithm regarding the creation of B-spline curves from the level-set function will be presented with some numerical examples in out talk.

\section{REFERENCES}

[1] T. Takahashi, T. Yamamoto, Y. Shimba, H. Isakari, T. Matsumoto, A framework of shape optimisation based on the isogeometric boundary element method toward designing thin-silicon photovoltaic devices, Engineering with Computers 35 (2) 423-449 (2019).

[2] G. H. Liu, Y. S. Wong, Y. F. Zhang, H. T. Loh, Adaptive fairing of digitized point data with discrete curvature, Computer Aided Design 34 (4) 309-320 .

[3] W. Zheng, P. Bo, Y. Liu, W. Wang, Fast b-spline curve fitting by l-bfgs, Computer Aided Geometric Design 29 (7) 448-462 (2012). 